\title{
Magnetically Levitated Linear Drive with Repulsive Magnetic Guidance
}

\author{
Markus Raab*, Bernd Gundelsweiler, Wolfgang Schinköthe \\ Institute of Design and Production in Precision Engineering, University Stuttgart, Stuttgart, Germany \\ Email address: \\ Markus.raab@ikff.uni-stuttgart.de (M. Raab) \\ ${ }^{*}$ Corresponding author
}

\section{To cite this article:}

Markus Raab, Bernd Gundelsweiler, Wolfgang Schinköthe. Magnetically Levitated Linear Drive with Repulsive Magnetic Guidance. International Journal of Mechanical Engineering and Applications. Vol. 7, No. 1, 2019, pp. 17-25. doi: 10.11648/j.ijmea.20190701.13

Received: February 15, 2019; Accepted: March 28, 2019; Published: April 18, 2019

\begin{abstract}
Linear direct drives are often used when high performance is required, because of their high dynamic and their good position accuracy. Usually direct drives are used with linear rolling guidance. In clean room and vacuum applications linear rolling guidance cannot be used as particles can be cause problems. In this paper a magnetically levitated linear direct drive with a combination of repulsive permanent magnet stabilization and Lorentz force based stabilization is presented. With the joint use of magnet fields high dynamic can be achieved in combination with a cost efficient hardware. Due to the use of repulsive permanent magnet forces, it is possible to levitate an armature with nearly no power dissipation. An ad-hoc control reduces the power dissipation to a value less than $10 \mathrm{~mW}$.
\end{abstract}

Keywords: Magnetic Levitation, Linear Direct Drive, Zero Power Levitation

\section{Introduction}

Due to their high dynamics, as well as their high precision and positioning accuracy, linear direct drives are increasingly used in various applications. An important aspect in the dimensioning of linear direct drives is the guidance of the armature, since this has a direct influence on the dynamics, positioning accuracy and operating conditions of the linear drive. Mechanical rolling elements and sliding guides form the standard because of their robustness, the low space requirement and an easy handling. A disadvantage, however, is the friction of the guide, which makes a high-precision positioning as well as a use under extreme conditions, such as for example in the high vacuum, impossible. These disadvantages can be compensated by the use of magnetic levitation guides.

The most widely used principle of magnetic levitation in precision engineering is the use of electromagnetic actuators (reluctance actuators), with which 5 DOF (degrees of freedom) of the guidance can be actively controlled [1-4]. The remaining sixth degree of freedom is the feed axis of the linear direct drive, which can be positioned by the linear direct drive itself. As described in Reutzsch and Gloess, magnetic levitation guides can also be developed on the basis of the Lorentz force $[4,5]$. By introducing coils into magnetic circuits, it is also possible to actively control 5 DOFs.

A disadvantage of these concepts, however, is the great expenditure on sensor technology, power electronics and computing power of a microcontroller in order to detect and actively influence the six DOF of an armature. Furthermore, complex control algorithms are required for this purpose. For this reason, it is often appropriate to passively stabilize degrees of freedom, which are less critical for the respective application. This can be done by the use of permanent magnetic bearings (guides) on the basis of attractive and repulsive magnetic forces.

In permanent-magnetic bearings, repulsive magnetic forces are often used [6-9]. If, as shown in Figure 1, a free-moving ring magnet is inserted between two statically mounted permanent magnets and its polarity is opposite to the fixed magnets, the magnets repel each other. As a result, the $\mathrm{X}$-axis of the magnetic levitation guide can be stabilized specifically with a positive stiffness (see A). However, a permanent-magnetic bearing always leads to a destabilization of other axes due to the Ernshaw theorem, which results in a negative stiffness in the destabilization of the $\mathrm{Y}$-axis (see B) [10]. For this reason, in a system with permanent-magnetic bearings, at least one degree of freedom must always actively compensate this negative spring stiffness.

The stabilization of the remaining degrees of freedom can be done in touch or without contact [11]. Most concepts consists in 
the use of position-controlled electromagnetic actuators, which are mounted at the corners of an armature. These actuators generate forces on the basis of Maxwell's tensile force between the actuators and a ferromagnetic return iron on the stator. By this configuration, the system is levitated in six degrees of freedom. A disadvantage, however, is that an additional magnetic circuit must be integrated by the electromagnets, which requires a back iron in the armature. Another concept for stabilization in the case of repulsive magnetic guidance is the use of multi-coordinate motors, which enable an active regulation of three degrees of freedom [7].

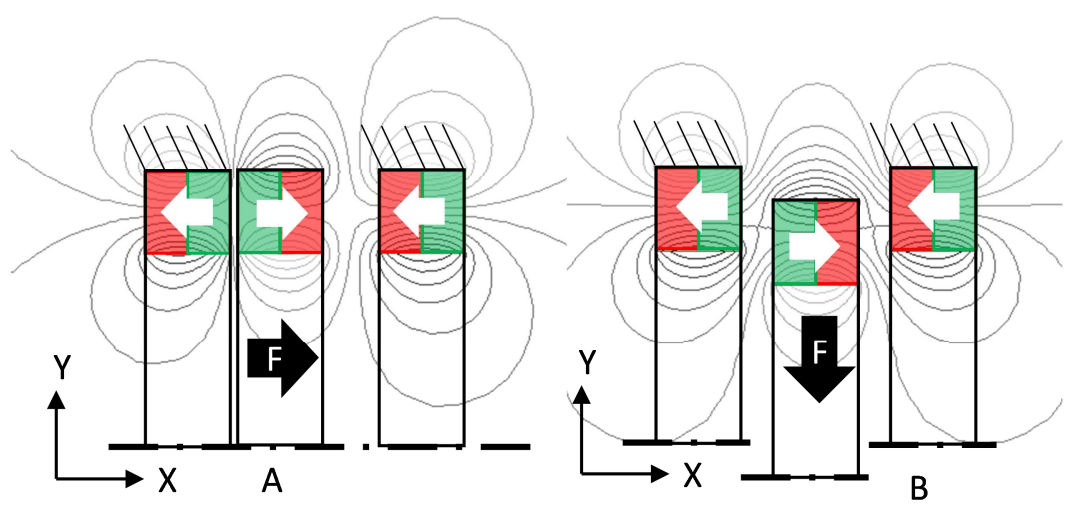

Figure 1. Repulsive magnet configuration.

In this paper, a magnetic levitation guidance for linear direct drives in precision engineering is presented based on permanent-magnetic levitation guidance in combination with a stabilization by electrodynamic Lorentz coils. By combining these two technologies, a function-integrated use of both effects is possible.

Section 2 shows the motor and guidance concept. The development of the motor axis is shown in section 3 . In section 4 the design and modeling of the magnetic guidance is described, which is followed by the control of the system in section 5 . The overall system is described in section 6 .

\section{General Concept}

The concept presented here uses permanent magnets to stabilize the vertical degrees of freedom $\left(Z, \varphi_{x}, \varphi_{Y}\right)$. Two permanent magnet tracks are mounted in the stator as shown in Figure 1. In the armature there are two oppositely polarized permanent magnets per track which act as guidance magnets. Due to the construction as shown in Figure 2, it is possible to provide the movement in the $\mathrm{Z}$ axis as well as the rotational degrees of freedom $\varphi_{x}$ and $\varphi_{y}$ with a positive stiffness.

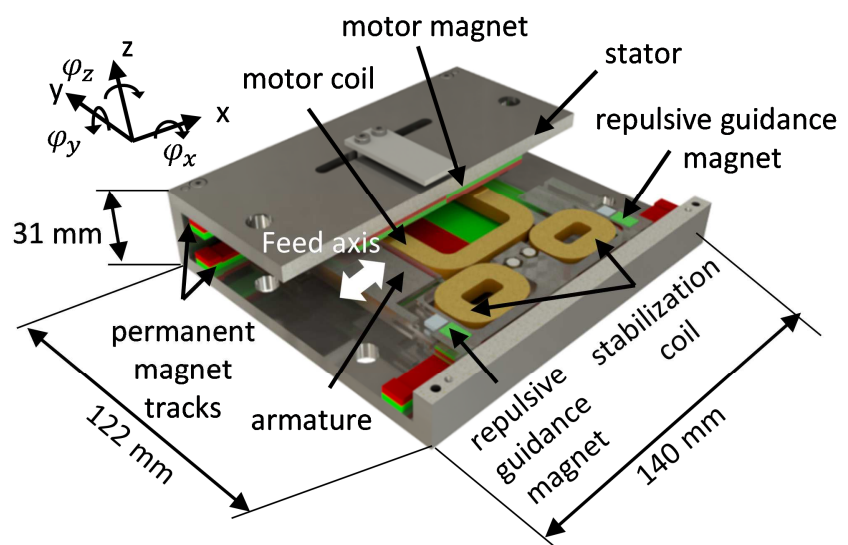

However, the permanent-magnetic bearings result in a destabilization of the horizontal degrees of freedom ( $\mathrm{Y}$ and $\varphi_{z}$ ). In order to be able to hold the armature in its middle levitated position, four stabilizing coils are mounted in the armature, which are also penetrated by the magnetic field of the permanent magnet tracks. By energizing the coils, forces can be generated in the destabilized axes and thus the armature can be held in its center position. In order to stabilize the armature, a measurement of the position as well as an active control of the destabilized degrees of freedom are necessary. For this purpose, Hall Elements are located as position sensors on the armature, which detect the $\mathrm{Y}-$ and $\varphi_{z}$ - axis and pass it on to a microcontroller. A homopolar flat coil drive with a travel distance of $30 \mathrm{~mm}$ is used as the drive of the feed axis (X).

\section{Motor Axis}

The feed axis of the magnetic levitation drive is designed as a homopolar flat coil drive with a moving coil. In this case, a constant, nearly homogeneous magnetic field is built up by oppositely poled permanent magnets, which are mounted in the stator. By using a coil which is located in the air gap between the magnets, a force can be exerted on the armature on the basis of the Lorentz force. A schematic view is shown in Figure 3.

Due to the linear relationship between the current through the coil and the output force, it is thus possible to selectively generate the forces of the drive. The force constant $k_{S X}$ which describes the ratio between current $I$ and force $F$ of the drive can be calculated using the following equation based on magnetic flux density $B$, length of the wire in the magnetic field $l$ and number of wires in the magnetic field $N$.

$$
k_{S X}=B \cdot l \cdot N \cdot 2=10,7 \mathrm{~N} / A
$$

Figure 2. Sectional view of the drive. 


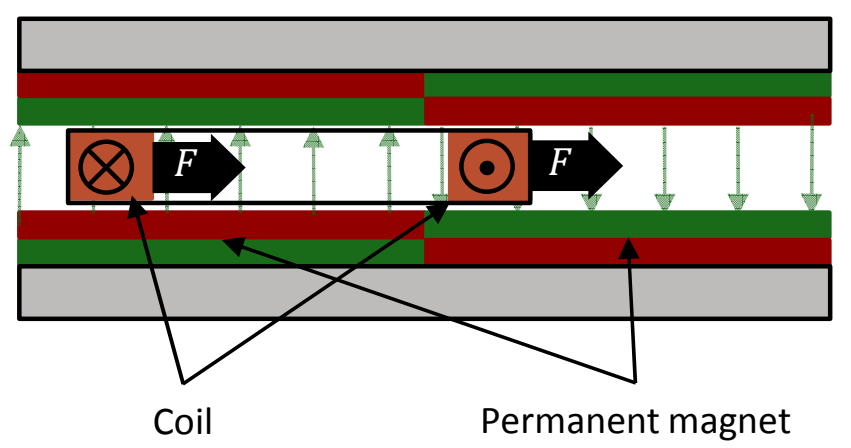

Figure 3. Schematic view of a homopolar linear drive.

The calculation of the force constant was carried out analytically in a first step and then finely tuned using FEM. The mass of the armature was determined both by CAD and experimentally and is $166 \mathrm{~g}$. The drive is operated in a controlled manner. For this purpose, an analog current source with a controller bandwidth of $2 \mathrm{kHz}$ is used. So, the speed-dependent induced voltage can be neglected. Due to the magnetic levitation, the friction on the armature are very low. The feed axis is modeled as a moving mass $m$ without friction. Thus the dynamics of the feed axis can be described with the following transfer function $G_{x}$.

$$
F_{x}=k_{S X} \cdot I=m \cdot \ddot{x} \rightarrow G_{x}=\frac{x}{I}=\frac{k_{S X}}{m \cdot s^{2}}
$$

Using the transfer function of (2), the Bode diagram of the magnetic levitation drive can be calculated in the X-axis. For this purpose the Bode diagram was calculated analytically in a first step. Finally, the Bode diagram of the real structure was measured. It can clearly be seen, that in a frequency range from 2 to $100 \mathrm{~Hz}$ there is a good correspondence between the models and the drive.

Only the mass of the armature and the force constant $\mathrm{k}_{\mathrm{SX}}$ have an influence on the behavior of the drive in the X-axis (see (2)). Since the mass could be determined by a scale, a good comparability between the model and the measurement can be established as it is depicted in Figure 4 and thus a good correspondence between the calculated and the actual force constant can be seen.
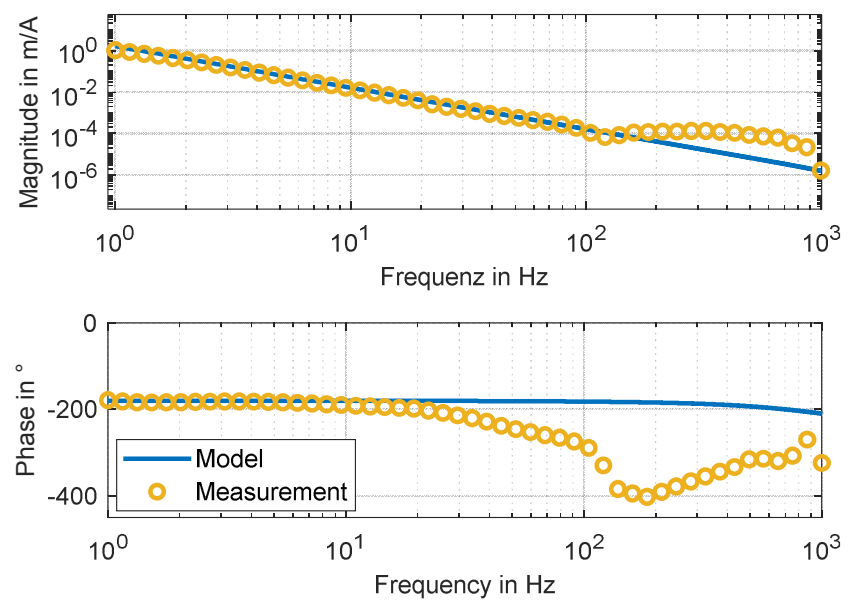

Figure 4. Simulation and measurement of the frequency response of the motor axis.

\section{Magnetic Guidance}

The aim of the magnetic levitation guidance is to achieve a high positive stiffness's in the passively stabilized axes of the drive taking into account the maximum force of the stabilizing coils as well as the maximum permissible dynamics of the system. To model this system, a simulation with Matlab ${ }^{\circledR}$ Simulink, in combination with values previously determined by FEM simulation using ANSYS Maxwell, is performed. In the following section we will discuss the design of the permanent magnet magnetic guides as well as the analytical modeling of the system. At the end of the section the used power electronics and sensing electronics of the guidance are described.

\subsection{Design of Passive Magnet Guides}

As described in general concept 2, the horizontal axes are stabilized by repulsive permanent magnetic forces. For this purpose, two permanent magnet tracks are mounted in the stator, and four oppositely poled magnets are located in the armature. This creates four bearing locations. If the armature and thus a guide is deflected in the Z-axis, a repelling force is created in this axis. There is a linear relationship between deflection and force which can be described by a spring constant $\mathrm{k}_{\mathrm{Z}}$. If the armature is deflected from its center position in the $\mathrm{Y}$-axis, a force is produced which forces the armature out of its center. The greater the deflection of the armature, the greater is the force generated by the permanent magnets. This characteristic is described by a negative stiffness $\mathrm{k}_{\mathrm{Y}}$ which is linear in the movement range of the drive. Both properties are summarized in the following Figure 5.

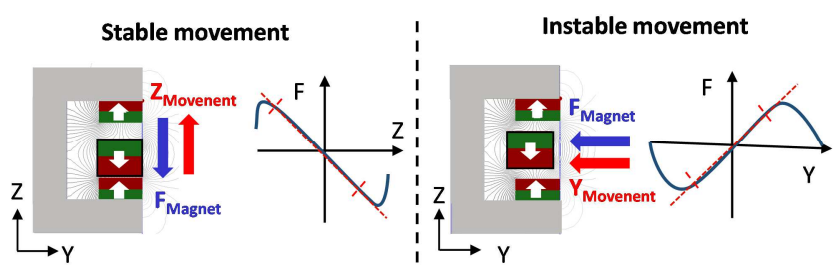

Figure 5. Stable and instable axis.

For the design of the permanent magnetic bearings, the resulting forces which arise during displacement of the armature from its zero position are determined in ANSYS Maxwell by an FEM simulation. By means of a parameter study, it is subsequently possible to select variants with a suitable ratio between high positive spring stiffness in the $\mathrm{Z}$-axis, as well as relatively low stiffness in the $\mathrm{Y}$-axis. On the basis of the stiffness of the individual bearings, it is possible to calculate the overall stiffness's of the drive.

This results in the relationships of (3) of the stiffness's of a bearing with respect to the guidance of the drive and the geometrical dependencies shown in Figure 6. The simulated stiffness's of the drive are given in the following equation. It can be seen that the negative stiffness of the $\mathrm{Y}$ axis is only slightly higher than the desired positive stiffness. Thus a good relationship between both stiffness's was achieved. 


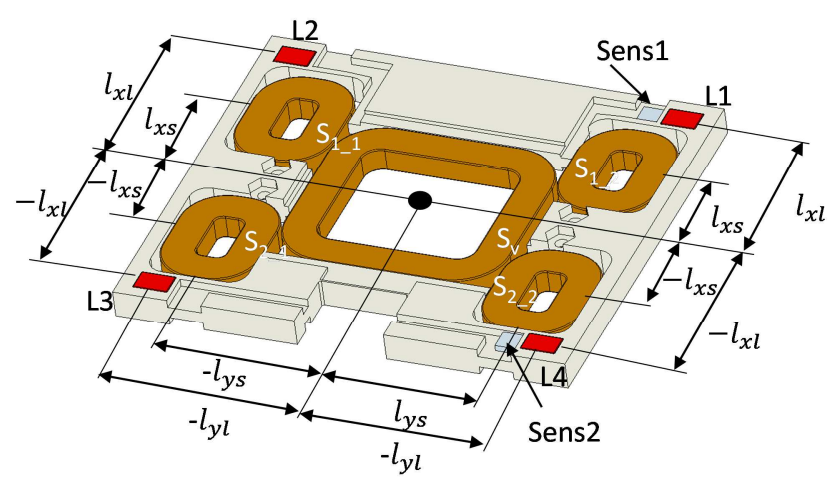

Figure 6. Geometrical dependencies.

$$
\begin{gathered}
k_{z}=4 \cdot k_{z_{\text {Lager }}}=10700 \mathrm{~N} / \mathrm{m} \\
(9865 \mathrm{~N} / \mathrm{m}-13200 \mathrm{~N} / \mathrm{m}) \\
k_{y}=4 \cdot k_{y_{\text {Lager }}}=-11500 \mathrm{~N} / \mathrm{m} \\
(-9473 \mathrm{~N} / \mathrm{m}-13860 \mathrm{~N} / \mathrm{m}) \\
k_{\varphi z}=4 \cdot l_{x l}^{2} \cdot k_{y_{\text {Lager }}}=-20.2 \mathrm{Nm} / \mathrm{rad} \\
(-16.4 \mathrm{Nm} / \mathrm{rad}-24.5 \mathrm{Nm} / \mathrm{rad})
\end{gathered}
$$

The remanence flux density $B_{\mathrm{r}}$ has a big influence on the achievable stiffnesses. This is indicated by manufacturers with a tolerance of $\pm 10 \%$. For this reason, a tolerance range must always be specified for a simulation which is attached to the above results. The comparison with the simulated stiffness is shown in Table 1 . It can be seen that an approximately linear relationship exists for all measured stiffness's. The negative stiffness of the drive is also within the tolerance range of the simulation. Only the measured Z-stiffness is slightly below the expected value.

Table 1. Comparison of the measured and simulated Stiffness's.

\begin{tabular}{l}
\hline Stiffness $\mathbf{Z}$ axis \\
Slightly lower than expacted
\end{tabular}

In contrast to the high stiffness's in the passive stabilized axes, only very small forces are to occur in the $\mathrm{X}$-axis (motor axis). The following Figure 7 shows the forces occurring during the movement of the armature in the X-axis. As can be seen, the parasitic forces are low in the middle travel range of the drive. The force increases only at the edge areas ( $15 \mathrm{~mm}$ to $10 \mathrm{~mm}$ ). If this effect is still too strong for a particular application, it can be reduced by longer permanent magnet tracks.

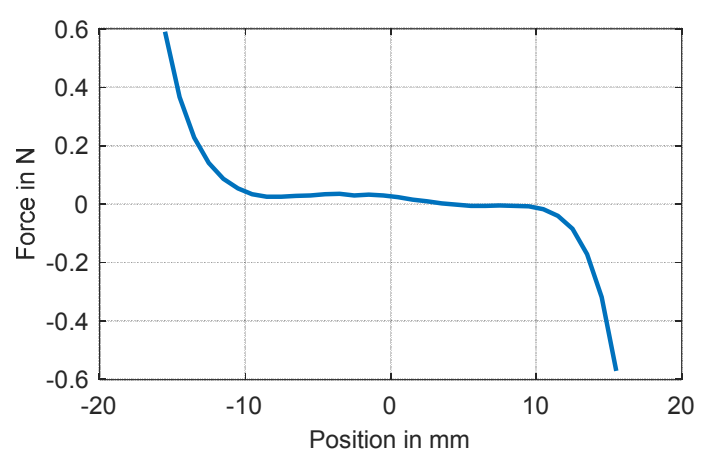

Figure 7. Measured stiffness of the X axis.

In addition to the stiffness in the individual axes, the damping of the passive stabilized axles is an important criterion, since this cannot be directly influenced. For this reason, the damping in the Z-axis is determined. A damping of $5.08 \mathrm{Ns} / \mathrm{m}$ which corresponds to a logarithmic decrement of 0.4 is measured on the basis of the measurement shown in Figure 8. Furthermore, it is possible to determine the Z-stiffness by means of the oscillation frequency and the mass of the armature. This results in a value of $8565 \mathrm{~N} / \mathrm{m}$, which is slightly less than the previously determined stiffness for this axis.

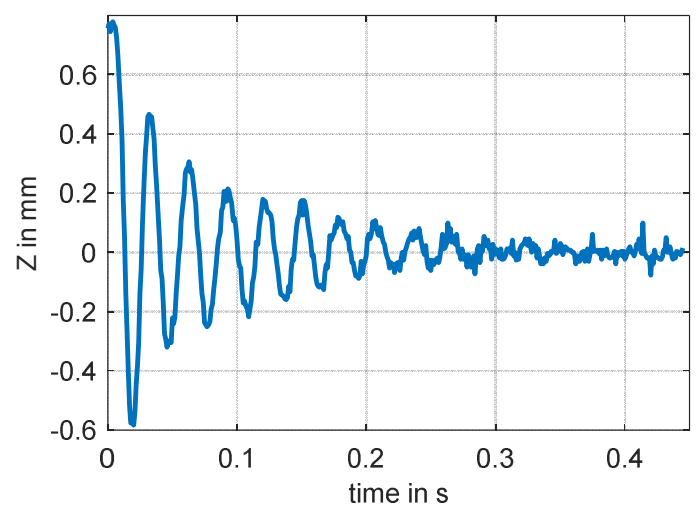

Figure 8. Damping of the $Z$ axis.

\subsection{Modelling and Design of the Electromagnetic System}

The following section describes the modeling and design of the electromechanical system. The aim is to determine the relationships between the currents of the individual actuators and the position of the armature. If the stabilizing coils S1 and S2 (see Figure 2 and Figure 6) are energized the same, forces are generated in the Y-axis. Because of the Lorentz force, there is a linear relationship between current and force, which is described by the constant $\mathrm{k}_{\mathrm{SY}}$. The geometrical relationships of Figure 6 also allow to determine the achievable stabilizing moments which arise, when the coils S1 and S2 are energized oppositely. In this case, the lengths $1_{\mathrm{xs}}$ and $1_{\mathrm{xl}}$ represent the distance from the center of the armature to the center of the stabilizing coil in the X-direction, as well as the distance from the center point of the armature to the center point of the moving bearing magnet. The force relationship between coil current and position of the rotor is shown in the following equation. 


$$
\left(\begin{array}{c}
F_{y} \\
M_{z}
\end{array}\right)=k_{S Y} \cdot\left(\begin{array}{cc}
1 & 1 \\
l_{x S} & -l_{x S}
\end{array}\right) \cdot\left(\begin{array}{l}
I_{s 1} \\
I_{s 2}
\end{array}\right)+k_{Y} \cdot\left(\begin{array}{cc}
2 & 0 \\
0 & 2 \cdot l_{x l}^{2}
\end{array}\right) \cdot\left(\begin{array}{c}
y \\
\varphi z
\end{array}\right)
$$

Thus, linear transfer functions can be calculated for the two axes of the magnetic levitation guide to be controlled (5), the masses and moments of inertia of the armature being determined in advance using a CAD model. It can be seen from the equations that it is a second order system with a spring stiffness.

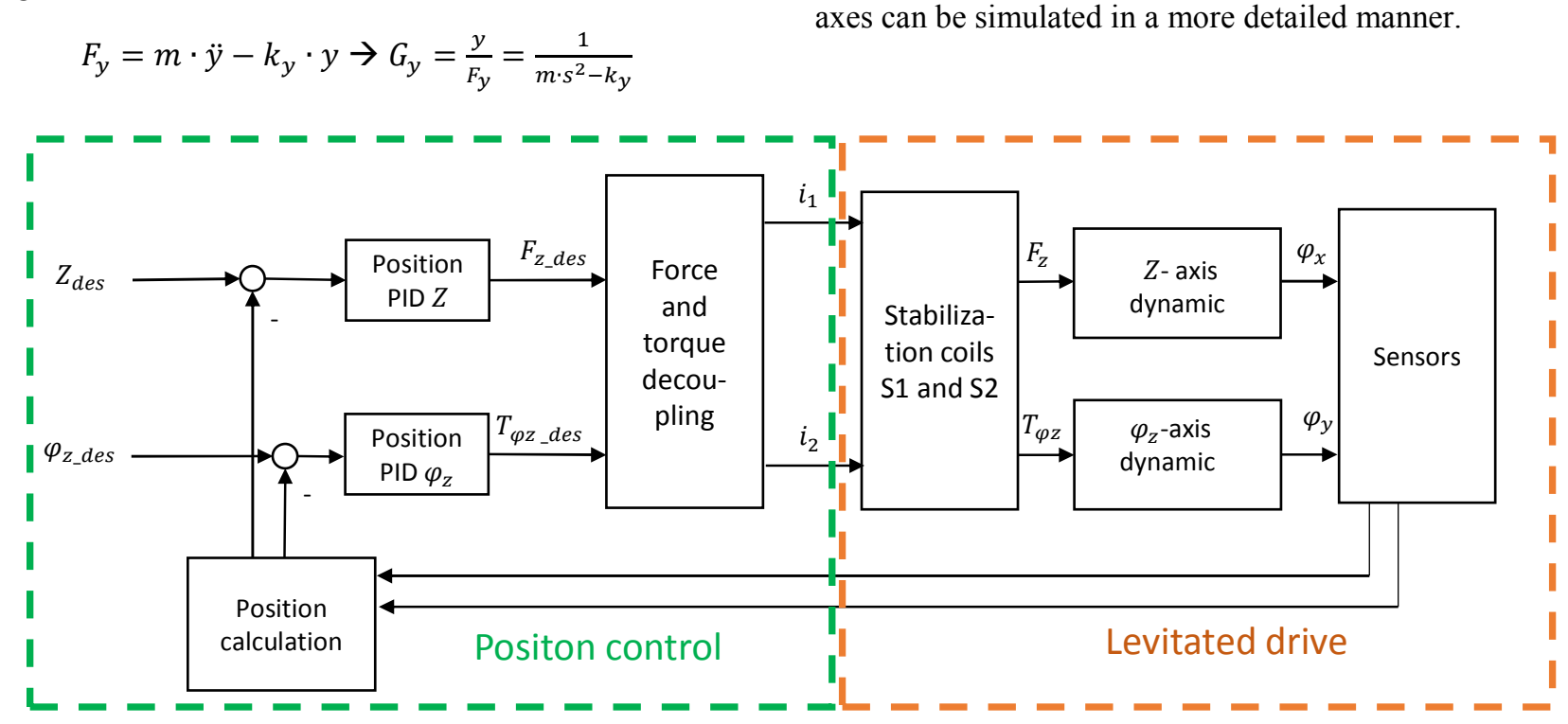

Figure 9. Position control and motor model.
In order to verify the calculated dynamics of the $\mathrm{Y}$ - and $\varphi_{z}$-axes, it is possible to calculate the Bode diagramm from (5) analytically. Furthermore, the Bode plot can be simulated for the numerical model using an analysis script and measured in the actual setup. The Bode plot of the Y-axis is shown in constant up to a frequency of approx. $30 \mathrm{~Hz}$ and then decreases with a slope of -2 decades per decade. The phase of the system is approximately $-180^{\circ}$. At higher frequencies, a drop in the phase can be detected here by a sampling time of $100 \mu \mathrm{s}$ and by a current control of $2 \mathrm{kHz}$.
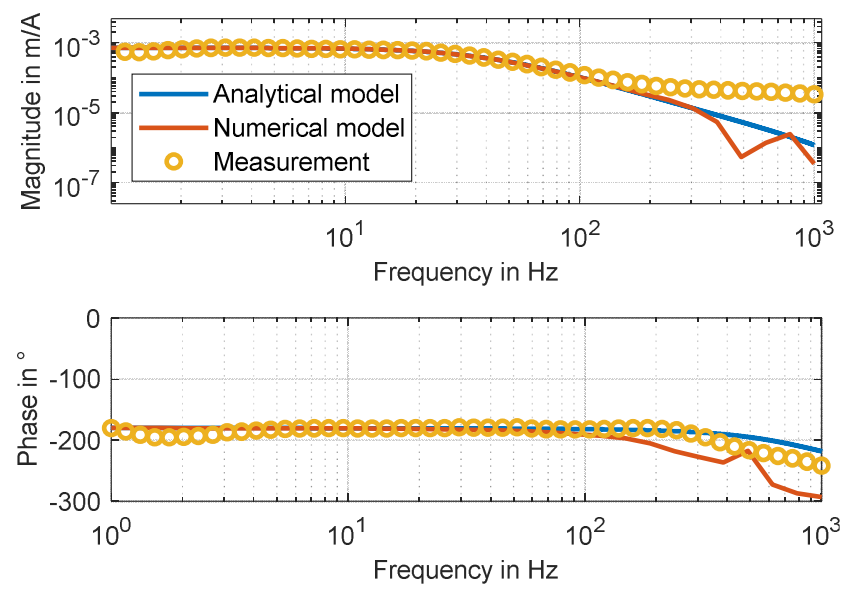

Figure 10. Measured and simulated Bode plot of the Y-axis.

The comparison between the measurement of the Bode plot, Figure 10. It can be seen that the amplitude response is

$$
M_{z}=J_{z} \cdot \ddot{\varphi}_{z}-k_{\varphi z} \cdot \varphi_{z} \rightarrow G_{\varphi z}=\frac{\varphi z}{M_{z}}=\frac{1}{J_{z} \cdot s^{2}-k_{\varphi z}}
$$

In addition to the purely analytical view of the drive, it is possible to design a numerical model, which also takes into account nonlinear effects such as current limitation and conversions between different coordinates. The model is shown in Figure 9. On the basis of this model, required currents, frequency response and stability of the individual axes can be simulated in a more detailed manner. the analytical model (5) and the numerical model shows a good comparability for the $\mathrm{Y}$-axis (Figure 10). Only at frequencies higher than $200 \mathrm{~Hz}$ the measurement deviates from the model predictions due to measuring noise and the magnetic field of the coils to the sensor system.

When looking at the Bode plot of the $\varphi_{z}$-axis (Figure 11), it is noticeable that the measured curve is approximately $15 \%$ above the analytically and numerically determined values. The reason for this is the difference between measured and simulated negative stiffness in this axis (see Table 1) and greater uncertainties with respect to the mass inertia given by the CAD system in this axis.
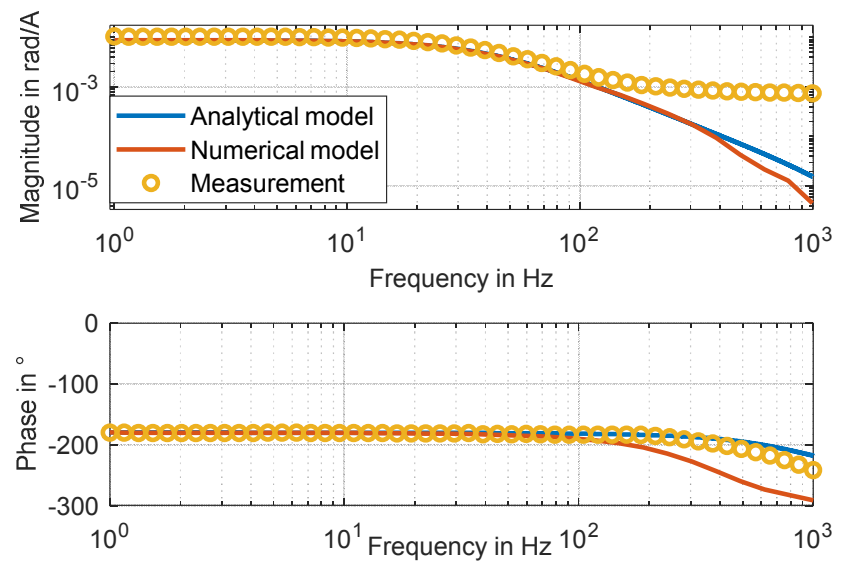

Figure 11. Measured and simulated frequency response of the $\varphi_{z}$-axis. 


\subsection{Power Electronics}

To control the three coils (feed coil, coil pair S1, coil pair S2), currents of up to $3 \mathrm{~A}$ are required. In order to keep the time constant between control signal and current through the coil low, the use of a current control loop is recommended. To implement this in the drive, an analog current control board with a control bandwidth of $2 \mathrm{kHz}$ is used. By using analog signals, EMC disturbance which would result from PWM control are kept low. A further advantage of this control is the clear connection between the control signal and the current. This allows good information about the behavior in the drive

Nevertheless, the analog amplifiers are bigger in space than pulse with modulation (PWM) controllers. If the space of the amplifiers is limited, amplifiers using PWM can also be used.

\subsection{Position Detection}

Three sensors are required to determine the position of the armature. The motor axis is determined by a laser triangulation sensor with a measurement range of $50 \mathrm{~mm}$ and an accuracy of some $\mu \mathrm{m}$.

For the detection of the axes $\mathrm{Y}$ and $\varphi_{z}$, Hall sensors are used which detect the magnetic field of the permanent magnet tracks. The positioning of the sensors is shown in Figure 12. For the best possible detection of the position of both axes, both sensors are placed as far away from each other as possible. The calculation of Cartesian coordinates calculation on the basis of the sensor values is given in (6). Where $1_{s}$ is the distance from the center of the Hall sensor Sens1 and Sens2 to the center of rotation of the armature.

$$
\left(\begin{array}{c}
Y \\
\varphi_{z}
\end{array}\right)=\left(\begin{array}{cc}
0.5 & 0.5 \\
\frac{1}{2 \cdot l_{s}} & \frac{-1}{2 \cdot l_{s}}
\end{array}\right) \cdot\left(\begin{array}{l}
\text { Sens } 1 \\
\text { Sens } 2
\end{array}\right)
$$

As can be seen in Figure 12, the flux density of the magnetic field between the permanent magnet tracks is slightly curved. This effect would lead to measurement errors of the position, when position change of the armature in the Z-axis occurs. To reduce this effect, two sensors are used one position (see Figure 12) and their measurement signal is added. If the armature moves in the Z-axis, the flux density of a sensor increases and the flux density of the second sensor decreases which compensates errors.

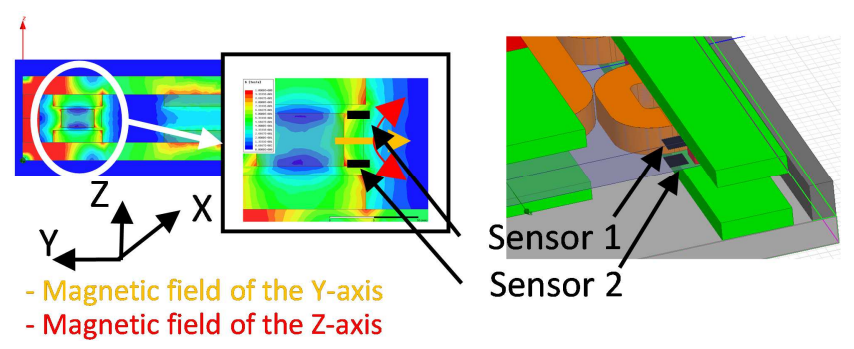

Figure 12. Magnetic field distribution.

\section{Control}

For the three axes $\mathrm{X}, \mathrm{Y}$ and $\varphi_{z}$ to be controlled, three decoupled PID-T1 controllers are designed. Thus, the magnetic levitation guide can be controlled as three mutually independent systems. The following section analyzes the stability of the control in the time and frequency domain. Furthermore, further filter methods and regulation for minimizing overshooting and hovering are presented.

In a last step, a control method to minimize the required power of levitation is presented, which reduces the power losses for stationary levitation down to some $\mathrm{mW}$.

\subsection{Basic Control Structure}

By analyzing the linear drive it can be seen, that the motor axis (X-axis) is not influenced by the other two controlled axes and does not affect them. For this reason, it can be driven and controlled as any conventional linear motor.

The two controlled axes of the linear guidance using individual PID-T1 controller, which calculates the electric currents needed to stabilize the guidance. These values are feed into a decoupling matrix, which is derived from (4) to the following equation. This way the two axis can be analyzed independently.

$$
\left(\begin{array}{l}
I_{S 1} \\
I_{S 2}
\end{array}\right)=\left(\begin{array}{cc}
\frac{1}{2} & \frac{1}{2 \cdot l_{x s}} \\
\frac{1}{2} & -\frac{1}{2 \cdot l_{x s}}
\end{array}\right) \cdot\left(\begin{array}{c}
F_{Z_{-} d e s} \\
T_{\varphi Z_{-} d e s}
\end{array}\right)
$$

From the Bode plot of the Y- and $\varphi_{z}$-axis the required control bandwidth can be derived to a frequency between 60 $\mathrm{Hz}$ and $100 \mathrm{~Hz}$. So a control bandwidth of $75 \mathrm{~Hz}$ has been chosen.

\subsection{Analysis of the Return Ratio}

To analyze the stability of the three control loops, the frequency response of the return ratio is determined for each axis. Based on this bode plot, it is possible to determine the achieved bandwidth of the control as well as amplitude and phase reserve.
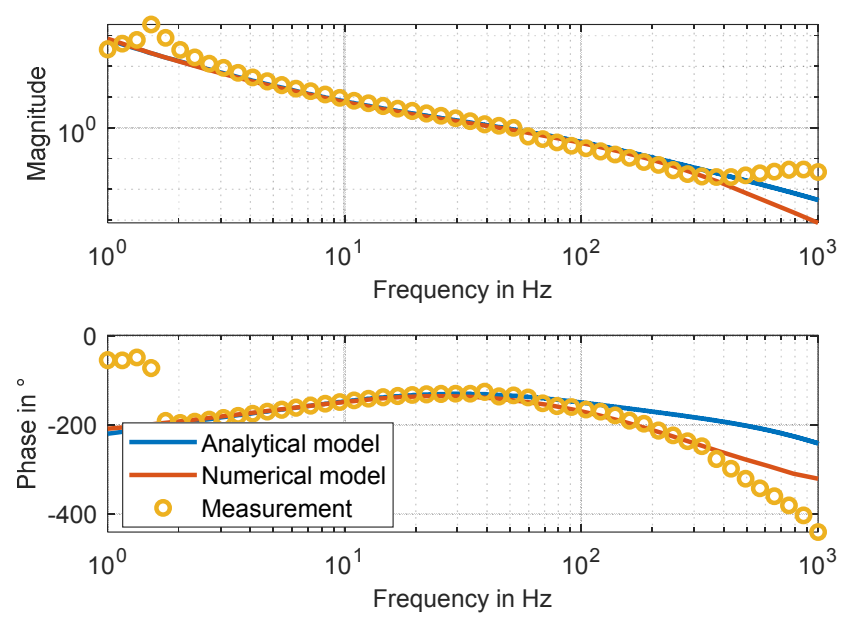

Figure 13. Return ratio of the $X$-axis.

The return ratio is calculated both analytically, as well as simulated in the numerical system for the controller design. In 
a next step the return ratio of the $\mathrm{X}$-axis is measured in the real setup as shown in Figure 13. The system bandwidth is $52 \mathrm{~Hz}$, which is somewhat higher than the desired $45 \mathrm{~Hz}$ bandwidth. The measured phase margin of the system in the $\mathrm{X}$-axis is $45^{\circ}$ and is only slightly lower than the expected phase margin of $48^{\circ}$. It can be seen that this axis stably adjusted.

Furthermore, the Bode plot of the return ration for the $\mathrm{Y}$-axis is simulated and measured as it is shown in Figure 14. A good comparability between model and measurement up to $100 \mathrm{~Hz}$ can be seen. The Y-axis control bandwidth is somewhat higher than the $68 \mathrm{~Hz}$ target bandwidth. The phase margin of the system in the $\mathrm{Y}$-axis is $30^{\circ}$ and is thus slightly higher than the desired phase margin of $28^{\circ}$. Thus the control is stably adjusted in the Y-axis. Comparable results are also measured in the $\varphi_{z}$-axis which are not shown here.
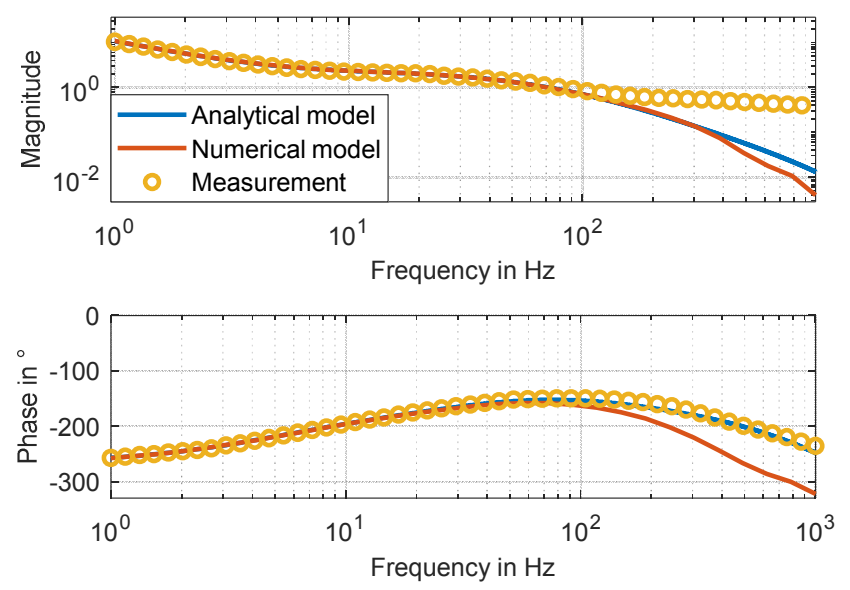

Figure 14. Return Ratio of the Y-axis.

\subsection{Step Responses of the Controlled System}
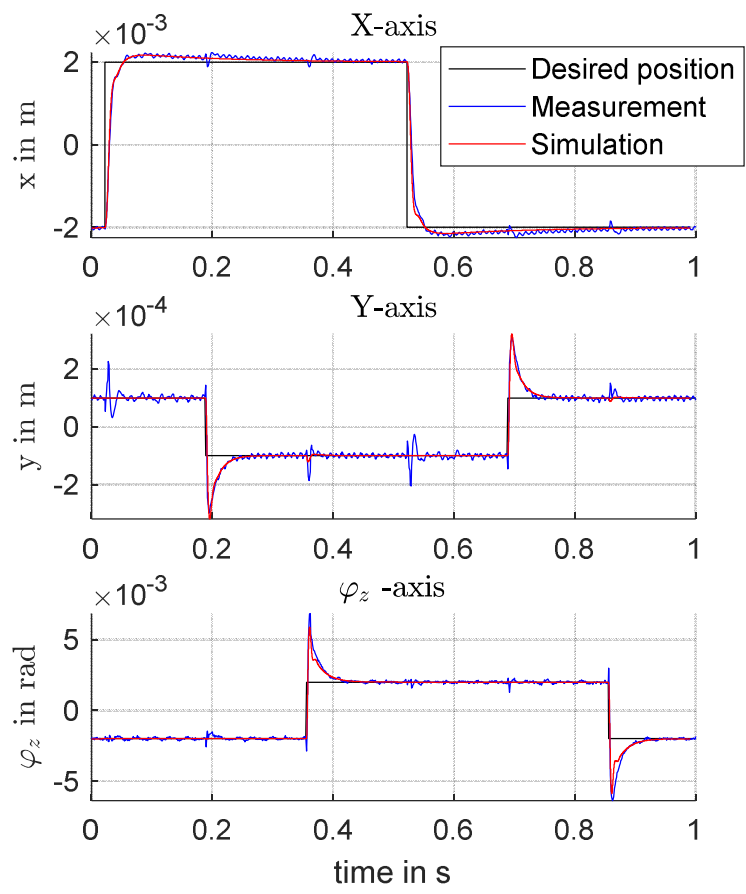

Figure 15. Step responses of the motor and guidance.
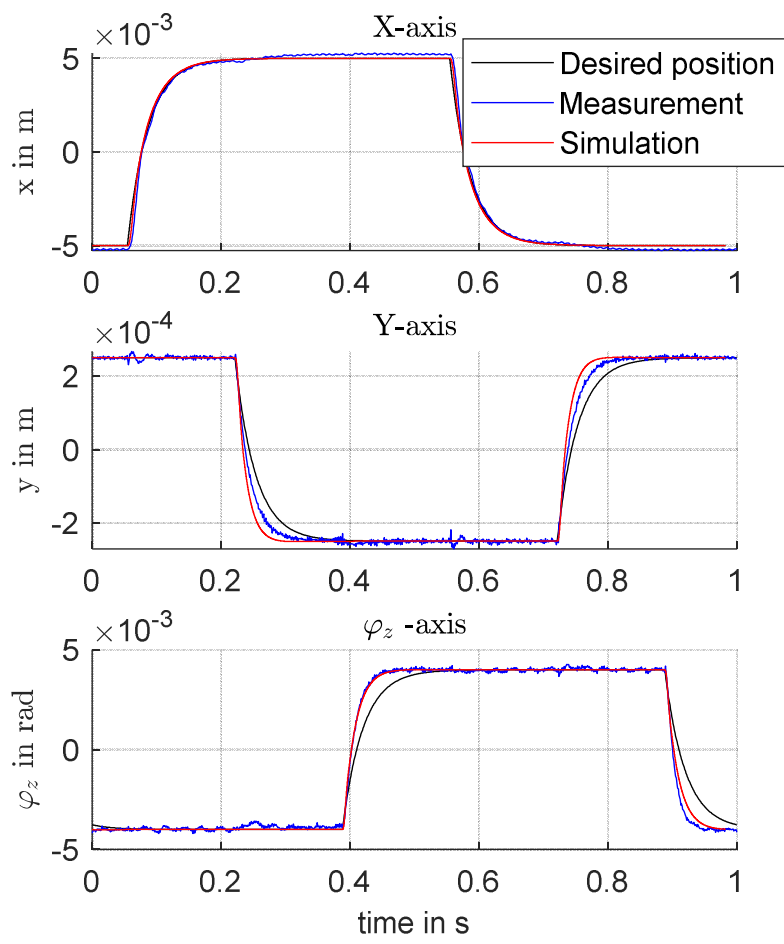

Figure 16. Step responses of the motor and guidance using an input filter.

In a next step, the step responses of the drive in the three controlled axes $\left(\mathrm{X}, \mathrm{Y}\right.$ and $\left.\varphi_{z}\right)$ are determined and compared with the simulated behavoir. The Figure 15 und 16 show step responses excitation in the three axes.

It can be seen, that there is a good correspondence between measurement and model. However, the overshoot in the positioning of an axis is clearly visible in Figure 15. This behavior can be reduced by a pre-filter of the desired signal that corresponds to a path planning, as shown in Figure 16. It can be seen that the coupling between different axes can thus be further reduced.

\subsection{Control for Minimum Levitation Power}

In the following, a controller structure for the power-minimized levitation with the linear direct drive is presented. Due to the negative stiffness in the $\mathrm{Y}$ - and $\varphi_{z}$-axis, deflections from the center position result in forces which need be used to compensate by forces of the stabilization coils.

The aim of a power-minimized control is the reduction of the coil current. This is achieved, when the control output of the position controllers of $\mathrm{Y}$ and $\varphi_{z}$ is zero, as these signals are direct proportional to the current through the coils and thus to the power losses of the guidance.

For this purpose, the control signal from the $\mathrm{Y}$ and $\varphi_{z}$ controllers is feed back to a cascaded PI controller as shown in Figure 17. This way the positon of the guidance is adjusted to the point where the minimum levitation power is required. On the other Hand, the possibility of specifying a specific $\mathrm{Y}$ and $\varphi_{z}$ position is, however, lost. 


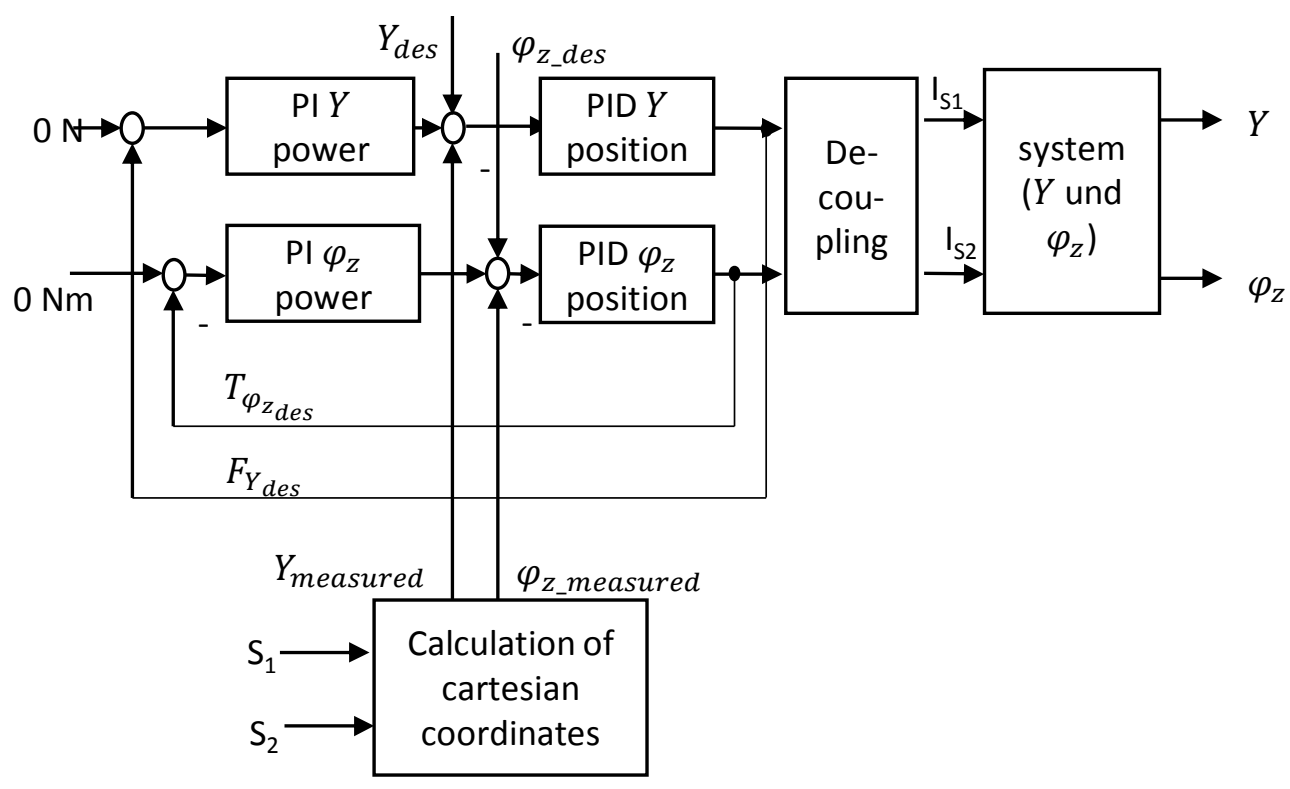

Figure 17. Control structure for minimum levitation power.

On the basis of this control, the drive was put into operation as shown in Figure 18. At the time of 2.3 seconds, the power-minimizing control was switched on. At the start time, the current through the coils is $0.5 \mathrm{~A}$ each, which corresponds to a total power of $3 \mathrm{~W}$. As can be seen, the control adjusts the position of the armature and thus minimizes the necessary levitation power. In the steady state, the required power to float is only $10 \mathrm{~mW}$. This value could be significantly reduced by noise-free sensor technology
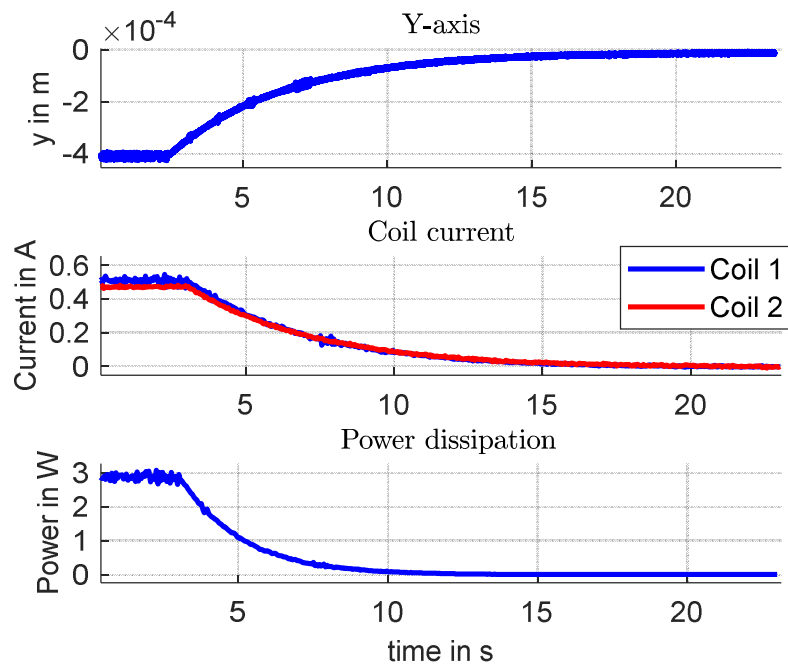

Figure 18. Zero power loss control.

\section{Overall System}

The following two figures show the overall design of the drive. The power electronics as well as the sensor PCB for evaluating the sound signal values can be seen in the upper Figure. The connection between armature and stator is also shown by an FFC cable is shown in the second figure.

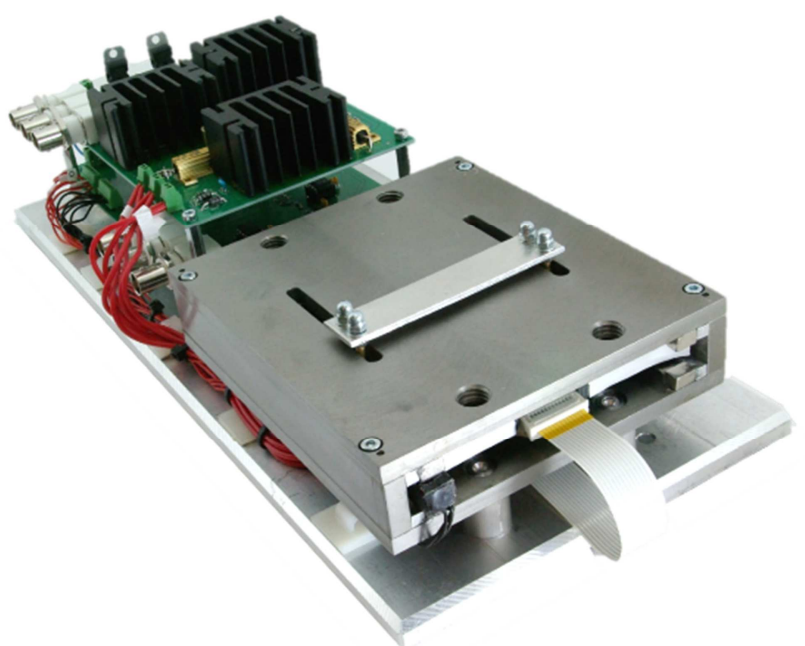

Figure 19. Picture of the levitated linear drive (side view).

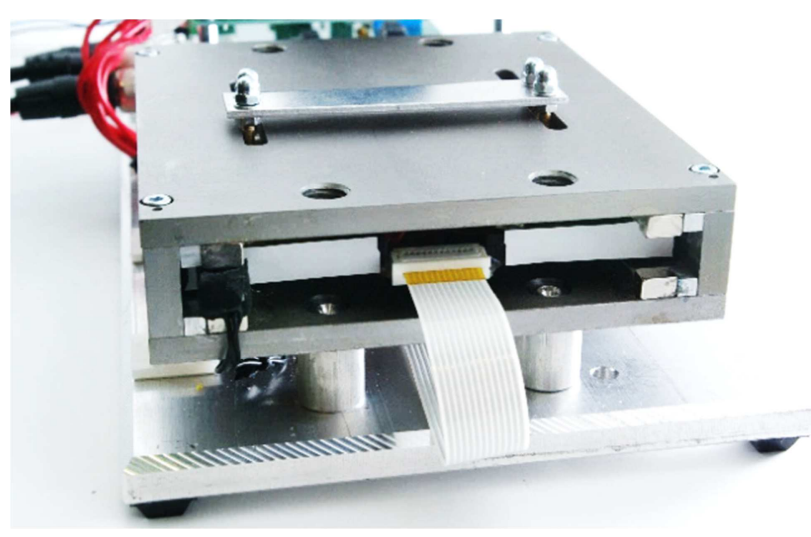

Figure 20. Picture of the levitated linear drive (front view).

\section{Conclusion}

The presented design shows a magnetically levitated linear drive with three permanent magnetically (passively) stabilized 
axes. The remaining guide axes are stabilized by the use of Lorentz coils and the magnetic field of the permanent magnet guides. The feed axis consists of a homopolar flat coil drive stiffnesses and forces of the drive were previously determined and optimized using FEM simulations. The measurements show a good agreement between the simulated and the measured values. By the use of an optimized controller structure, this design makes it possible to reduce the levitation power of the drive for varying weight loads between $0 \mathrm{~g}$ and $500 \mathrm{~g}$ to less than $10 \mathrm{~mW}$.

\section{References}

[1] L. Zhou and D. Trumper, "Magnetically levitated Linear Stage for In-vaccum Transportation Tasks" 33rd anual meeting of the American Society of Precision Engineering (ASPE), Las Vegas, Nevada, USA, 2018.

[2] M. Tomida, K. Oka, A. Harada and J. Lin, "3 phase Linear Actuator in Magnetically Lavitated Linear Slider with Non-contact Power Supply", in Proc. of the $16^{\text {th }}$ International Symposium on Magnetic Bearings (ISMB 16), Bejing, China, 2018.

[3] M. Tousain, R. Tousain and J. van Eijk, "Active Magnetic Bearing Controls Strategy and Calibration", in Proc. of the ASPE 2008 Spring Topical Meeting. Berkeley, California. USA, 2008 .

[4] B. Reutzsch, "Entwicklung feinwerktechnischer Magnetschwebeführungen“, Doctoral Thesis, University Stuttgart. Institute of Design and Production in Precision Engineering, Stuttgart, Germany 2015.
[5] R. Gloess, A. Goos, "Nanometer Resolution of Magnetic Leviation Stages for Planar and Linear Scan Applications", in Proc. Of the $16^{\text {th }}$ International Symposium on Magnetic Bearings (ISMB 16), 2018.

[6] G. Gianolio and S. Zanolli, "Repulsive Permanent Magnets Transportation System", in Proc. of the 17th international Conference on Magnetically Levitated Systems (Maglev 2002) MAGLEV. Lausanne, Switzerland. 2002.

[7] H. Nguyen, G. Angelis, E. Molenaar, F. Sahin, H. Stoutjesdijk and M. Steinbuch, „Magnetic levitation for precision Motion with only two off the shelf linear mtors and a novel passive magnetic bearing Design: Modelling and control callenges“, In Proc. of the 24th anual meeting of the American Society of Precision Engineering (ASPE). Monterey, California 2009.

[8] F. Profumo, A. Tenconi and G. Gianolio, "Parameters and Forces of a PM Linear Synchronous Motor with Magnetic Guides for Industrial Applications: Computed and Experimental Results" in Proc. of the IEEE Industry Applications Conference Thirty-Fifth IAS Annual Meeting, Rome, Italy 2000.

[9] F. Profumo, A. Tenconi, G. Gianolio and K. Gigliotti, "Design and performance evaluation of a PM linear synchronous motor with magnetic guides for industrial applications", in Proc. of the IEEE Industry Applications Conference Thirty-Fourth IAS Annual Meeting. Phoenix, Az., USA, 1999.

[10] S. Ernshaw, "On the nature of the molecular forces which regulate the constitution of the luminiferous ether", Transactions of the Cambridge Philosophical Society, 1839.

[11] P. Bundig, "Direct Linear Drives for the application in High Vacuum", in Proc. of the 51. International Scientific Colloquium, Internationales Wissenschaftliches Kolloquium. Ilmenau, Germany, 2006. 